\title{
Early urinary biomarkers of acute kidney injury in preterm infants
}

\author{
Mina Hanna', Patrick D. Brophy², Peter J. Giannone' ${ }^{1}$, Mandar S. Joshi', John A. Bauer ${ }^{1}$ and Satish RamachandraRao ${ }^{3,4}$
}

BACKGROUND: Acute kidney injury (AKI) in the neonatal intensive care setting is multifactorial and is associated with significant morbidity and mortality. This study evaluates the utility of novel urinary biomarkers to predict the development and/or severity AKI in preterm infants.

METHODS: We performed a case-control study on a prospective cohort of preterm infants ( $<32 \mathrm{wk}$ ), to compare seven urine biomarkers between 25 infants with AKI and 20 infants without AKI.

RESULTS: Infants with AKI had significantly higher neutrophil gelatinase-associated lipocalin (NGAL) (median, control (CTRL) vs. AKl; 0.598 vs. $4.24 \mu \mathrm{g} / \mathrm{ml} ; P<0.0001)$. In contrast, urinary epidermal growth factor (EGF) levels were significantly lower in infants who developed AKI compared to controls (median, CTRL vs. AKl; 0.016 vs. $0.006 \mu \mathrm{g} / \mathrm{ml} ; P<0.001$ ). The area under the curve (AUC) for NGAL for prediction of stage I AKI on the day prior to AKI diagnosis (day-1) was 0.91, and for the prediction of stage II/III, AKI was 0.92. Similarly, urine EGF was a predictor of renal injury on day -1 (AUC: 0.97 for stage I and 0.86 for stage II/III AKI).

CONCLUSION: Urinary biomarkers may be useful to predict AKI development prior to changes in serum creatinine $(\mathrm{SCr})$ in preterm infants.

$\mathrm{n}$ recent decades, several advances in neonatal medicine has enhanced survival for preterm infants, most notably pulmonary surfactant and improved ventilation strategies. However, in most cases, the incidence of major morbidities has changed very little, especially for extremely premature infants $(1,2)$. Acute kidney injury (AKI), indicated by a rise in serum creatinine (SCr) occurs in approximately $12-18 \%$ of preterm neonates (3). Premature infants typically have immature renal structure and function at birth, are often exposed to nephrotoxic medications and are at high risk of AKI in the perinatal period. Very recent studies have demonstrated that the extrauterine environment is not optimal for kidney development, an effect which could be exaggerated in those with AKI (4). It has also been recognized that small changes in neonatal kidney function in this vulnerable population are associated with short-term consequences (fluid overload, increased length of stay, and death) (5-7). The role that AKI plays in the development of chronic kidney disease (CKD) in this population is still unknown. Few case reports document that CKD occurs in infants who had AKI; however, these studies are small singlecenter retrospective reports (8).

Current methodology identifying renal dysfunction is based upon serum creatinine and urine output (UOP). The shortcomings of SCr and UOP to define AKI are well documented including: (i) a lag between initial renal injury and creatinine rise, (ii) inability to differentiate specific site of renal injury, (iii) increasing unreliability of creatinine measurement with increasing degree of renal injury due to tubular secretion of creatinine, and (iv) Renal supportive therapy, when required, removes creatinine making prognostication difficult (9). In the neonatal population, use of SCr to diagnose AKI is even more complex since initial neonatal creatinine reflects maternal values, which decrease over time and the estimated creatinine clearance (eCCL) changes as growth and renal development occur. The lack of reliable biomarkers of early structural kidney injury results in an undesirable delay in the clinical diagnosis. Early diagnosis of AKI in preterm infants is extremely important. Therefore, identifying earlier biomarkers of AKI is needed in this vulnerable patient population.

While investigators have begun to focus on AKI in the Adult and Pediatric Intensive Care Units, there is a paucity of data in the area of Neonatal AKI, in which the population is heterogeneous at varying gestational ages and AKI etiology is often multifactorial $(10,11)$. Abitbol et al. (12) showed that serum cystatin $\mathrm{C}$ level is a superior biomarker to serum $\mathrm{Cr}$ in the assessment of GFR in a cross-sectional observational cohort of premature infants. The urine represents a rich, noninvasive source of potential biomarkers. This is particularly relevant in the neonatal population where blood-sparing studies are paramount to standard clinical care. To determine whether there is an association between candidate urinary biomarkers and AKI in this vulnerable population, we studied seven previously described urinary biomarkers: albumin,

'Department of Pediatrics, University of Kentucky College of Medicine, Lexington, Kentucky; ${ }^{2}$ Department of Pediatrics, University of lowa, lowa City, lowa; ${ }^{3} \mathrm{O}^{\prime}$ Brien Center for AKI Research, University of California-San Diego, San Diego, California; ${ }^{4}$ Department of Cell Biology and Molecular Genetics, Devaraj Urs Medical College, Kolar, India. Correspondence: Mina Hanna (mina.hanna@uky.edu)

Received 1 October 2015; accepted 2 February 2016; advance online publication 4 May 2016. doi:10.1038/pr.2016.70 
$\beta 2$-microglobulin ( $\beta 2 \mathrm{MG})$, cystatin C (Cys C), epidermal growth factor (EGF), neutrophil gelatinase-associated lipocalin (NGAL), osteopontin (OPN), and uromodulin (UMOD) $(13,14)$. Our aim was to determine whether urinary biomarkers concentrations increase before $\mathrm{SCr}$ elevation, and to evaluate the sensitivity and specificity of these biomarkers to predict the clinical course of AKI.

\section{RESULTS}

\section{Baseline Demographics and Biomarkers}

In the present study, 20 infants who never sustained AKI served as control (CTRL) and 25 infants who developed AKI based on the KDIGO classification served as cases. The average day of life in infants who developed AKI was 4 (range 2-7 d of life). Table 1 describes the maternal characteristics, infants' demographics, and clinical variables between those with and without AKI. No statistically significant differences in maternal demographic variables were seen between the

Table 1. Demographic data for infants with and without AKI

\begin{tabular}{|c|c|c|c|}
\hline & No AKI $(n=20)$ & $\operatorname{AKI}(n=25)$ & $P$ value \\
\hline \multicolumn{4}{|l|}{ Maternal characteristics } \\
\hline Age (mean, SD) & $24.4(2.7)$ & $23.4(4.6)$ & 0.47 \\
\hline Hypertension ( $n, \%)$ & $1(5 \%)$ & $2(8 \%)$ & 0.69 \\
\hline Pre-eclampsia $(n, \%)$ & $5(25 \%)$ & $5(20 \%)$ & 0.69 \\
\hline Drug use $(n, \%)$ & $3(15 \%)$ & $2(8 \%)$ & 0.46 \\
\hline Cigarette smoking $(n, \%)$ & $8(40 \%)$ & $7(28 \%)$ & 0.4 \\
\hline Antenatal steroid $(n, \%)$ & $15(75 \%)$ & $22(88 \%)$ & 0.26 \\
\hline Chorioamnionitis $(n, \%)$ & $0(0 \%)$ & $3(12 \%)$ & 0.11 \\
\hline Diabetes $(n, \%)$ & $2(10 \%)$ & $0(0 \%)$ & 0.11 \\
\hline Maternal antibiotics $(n, \%)$ & $0(0 \%)$ & $3(12 \%)$ & 0.11 \\
\hline Multiple gestation $(n, \%)$ & $6(30 \%)$ & $9(36 \%)$ & 0.68 \\
\hline Prenatal care $(n, \%)$ & $20(100 \%)$ & $24(96 \%)$ & 0.37 \\
\hline C-section $(n, \%)$ & $16(80 \%)$ & $15(60 \%)$ & 0.16 \\
\hline \multicolumn{4}{|l|}{ Infant characteristics } \\
\hline Birth weight (mean, SD;g) & $884.1(156.6)$ & $752.2(176.6)$ & 0.02 \\
\hline Gestational age (mean, SD; weeks) & $26(1.7)$ & $25.2(1.1)$ & 0.09 \\
\hline Male $(n, \%)$ & $8(40 \%)$ & $12(48 \%)$ & 0.6 \\
\hline Race $(n, \%)$ & & & 0.77 \\
\hline White & $18(90 \%)$ & $23(92 \%)$ & \\
\hline Black & $2(10 \%)$ & $2(8 \%)$ & \\
\hline \multicolumn{4}{|l|}{ Apgar score (median, IQ range) } \\
\hline $1 \mathrm{~min}$ & $5(2-6)$ & $2(1-4)$ & $<0.01$ \\
\hline $5 \min$ & $7(6-7)$ & $4(3-7)$ & 0.04 \\
\hline \multicolumn{4}{|l|}{ Clinical variables } \\
\hline Aminoglycoside $(n, \%)$ & $19(95 \%)$ & $23(92 \%)$ & 0.69 \\
\hline Indomethacin $(n, \%)$ & $7(35 \%)$ & $10(40 \%)$ & 0.73 \\
\hline Sepsis $(n, \%)$ & $3(15 \%)$ & $6(24 \%)$ & 0.46 \\
\hline $\operatorname{UAC}(n, \%)$ & $14(70 \%)$ & $23(92 \%)$ & 0.05 \\
\hline Pressors & $0(0 \%)$ & $9(36 \%)$ & $<0.05$ \\
\hline
\end{tabular}

AKI, acute kidney injury; UAC, umbilical arterial catheter. infants with $\mathrm{AKI}$ and those without $\mathrm{AKI}$, although no mothers of infants without AKI had chorioamnionitis, compared to 3 of 25 mothers $(12 \%)$ of infants with AKI $(P=0.32)$. Infants with AKI had lower birth weight (mean \pm SD; $752.2 \pm 176.6$ vs. $884.1 \pm 156.6 \mathrm{~g} ; P=0.02$ ), and lower Apgar score at 1 and $5 \mathrm{~min}$ than those without AKI. Otherwise, no major differences in clinical variables were detected between the two groups.

Additionally, we investigated the levels of urinary biomarkers in the control group across various gestational ages. As shown in Figure 1, there was no statistically significant difference between urinary biomarkers studied, across different gestational ages.

\section{Biomarkers Differences}

Figure 2 shows the differences of biomarkers values in infants with AKI and those without AKI, these biomarkers were analyzed on the day the injury initially occurred (day 0 ). We did not detect any statistically significant differences between control and AKI groups in the urinary levels of Albumin (Figure 2a) and $\beta 2 \mathrm{MG}$ (Figure 2b) $(P=\mathrm{NS})$. Infants with AKI had significantly higher urinary Cys $C$ levels (Figure 2c) (median CTRL vs. AKI; 0.98 vs. $6.09 \mu \mathrm{g} / \mathrm{ml} ; P<0.001$ ), and higher NGAL (Figure 2e) (median, CTRL vs. AKI; 0.598 vs. $4.24 \mu \mathrm{g} / \mathrm{ml} ; P<0.0001$ ). In contrast, urinary EGF levels (Figure 2d) were significantly lower in infants who developed AKI compared to controls (median, CTRL vs. AKI; 0.016 vs. $0.006 \mu \mathrm{g} / \mathrm{ml} ; P<0.001)$.

\section{Biomarkers as Early Predictors of AKI}

Further, we investigated the urinary levels of selected biomarkers on the day prior to AKI diagnosis (day -1). Urinary Albumin and $\beta 2 \mathrm{MG}$ levels did not differ on day -1 vs. day 0 . Urinary levels of Cys C, EGF, NGAL, OPN, and UMOD were at the same level as day 0 levels on day - 1 , these levels were significantly different from day matched control (no-AKI) group. Figure 3 demonstrates urine biomarkers levels for infants with AKI on the day they developed the renal injury (day 0) as well as the day before (day -1), and those without AKI. Table 2 shows the fold change of these biomarkers based on the values in day - 1 compared to the control group. Preterm infants with lower birth weight may possibly have higher baseline values of some urinary AKI biomarkers (12). Therefore, we incorporated birth weight into predictive models to control for this potential confounder. The area under the curve (AUC) for EGF, and NGAL for prediction of stage I AKI on day- 1 was 0.97 and 0.91 respectively (Figure 4), and for the prediction of stage II/III AKI was 0.86 and 0.92 respectively. The combination of EGF and NGAL was not significantly different in prediction of stage I AKI (AUC: 0.93) compared to EGF alone. However, the combined model using the two biomarkers improved the ability to predict stage II/III AKI compared to the models using NGAL or EGF alone (AUC: 0.95).

\section{DISCUSSION}

In this case-control study of AKI in preterm infants, we found that urinary biomarkers were associated with AKI, defined 


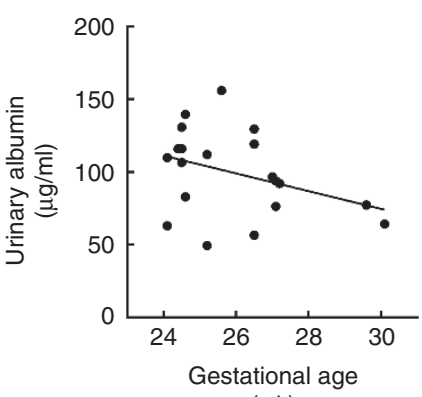

(wk)

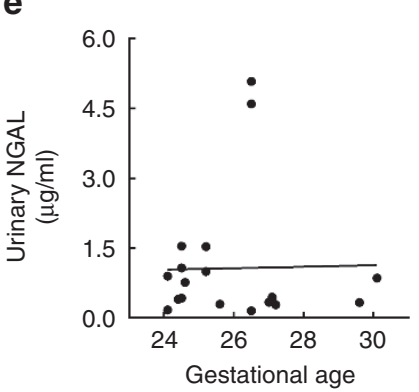

(wk)

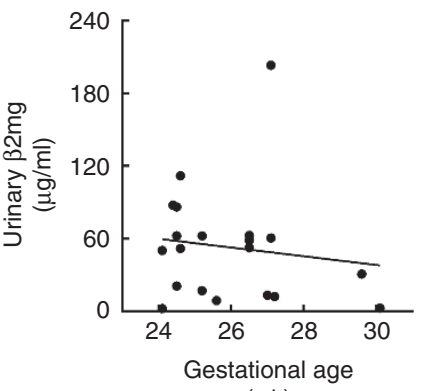

(wk)

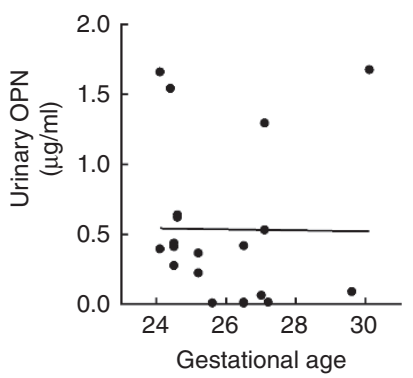

(wk)

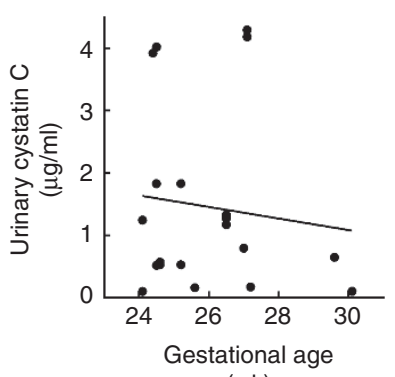

(wk)

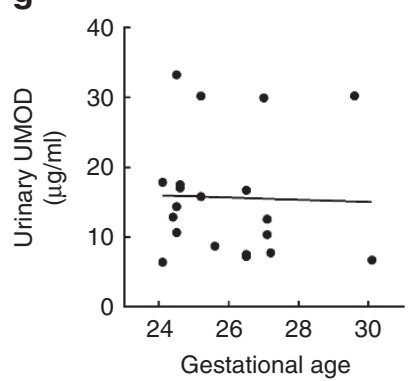

(wk)

g

Figure 1. Urine biomarkers levels in the control group at different gestational ages.

a

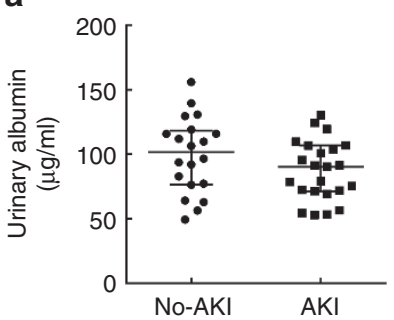

e

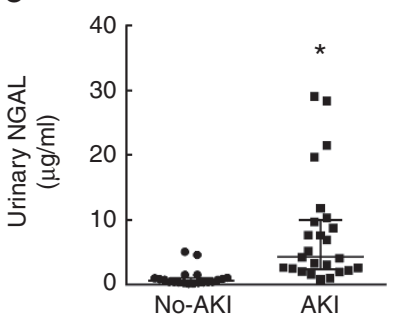

b

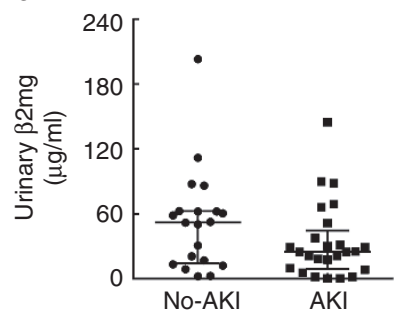

f

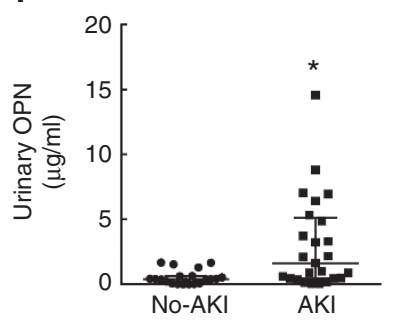

d

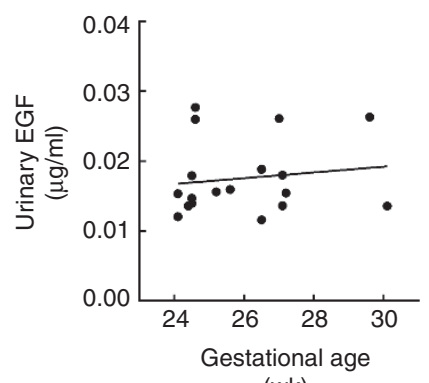

(wk)

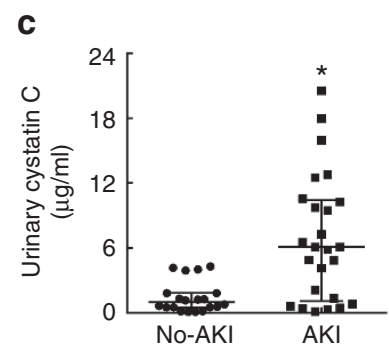

d

g

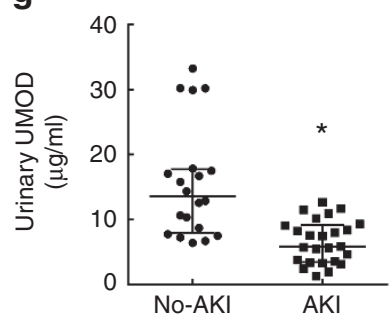

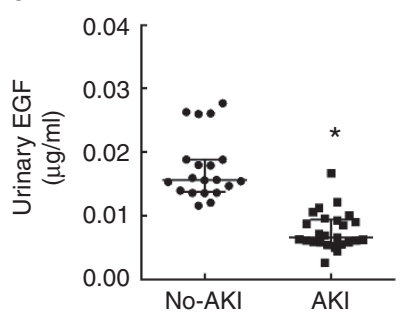

Figure 2. Variation of urinary biomarker levels in infants with acute kidney injury (AKI) and those without AKI. $\left({ }^{*} P<0.05\right)$.

based on the KDIGO classification of AKI. Urinary NGAL, OPN and Cys $\mathrm{C}$ increased significantly in infants who developed AKI, in contrast, urinary EGF and UMOD decreased significantly in this group. Urinary biomarkers demonstrated a significant change $24 \mathrm{~h}$ prior to contemporary creatininebased neonatal AKI definition. To our knowledge, this is the first study to demonstrate changes in these clinically relevant biomarkers on the day prior to AKI diagnosis in preterm infants. Early detection of renal injury will be highly valuable in altering clinical course of action and may prevent long term complications of AKI in these susceptible preterm infants.
Urine NGAL has been recently reported to be a useful early AKI marker that predicted development of severe AKI in a heterogeneous group of pediatric patients admitted to the PICU with unknown timing of kidney injury (15). A multicenter pooled analysis of prospective studies shows that NGAL can predict mortality even in the absence of diagnostic increases in serum creatinine (16). A recent pilot study showed that higher urinary NGAL concentrations were associated with the documentation of stage I AKI, but it failed to predict its development $1-2 \mathrm{~d}$ earlier in preterm infants (17). On the contrary, we found urine NGAL to be a good early marker of AKI development prior to serum creatinine rise in this cohort of 


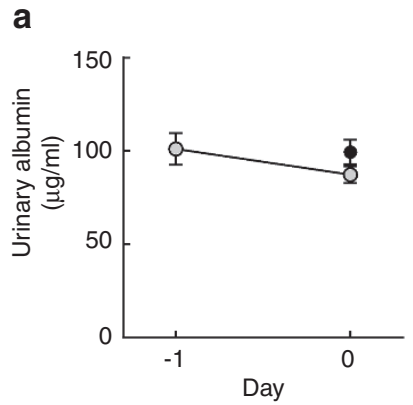

b
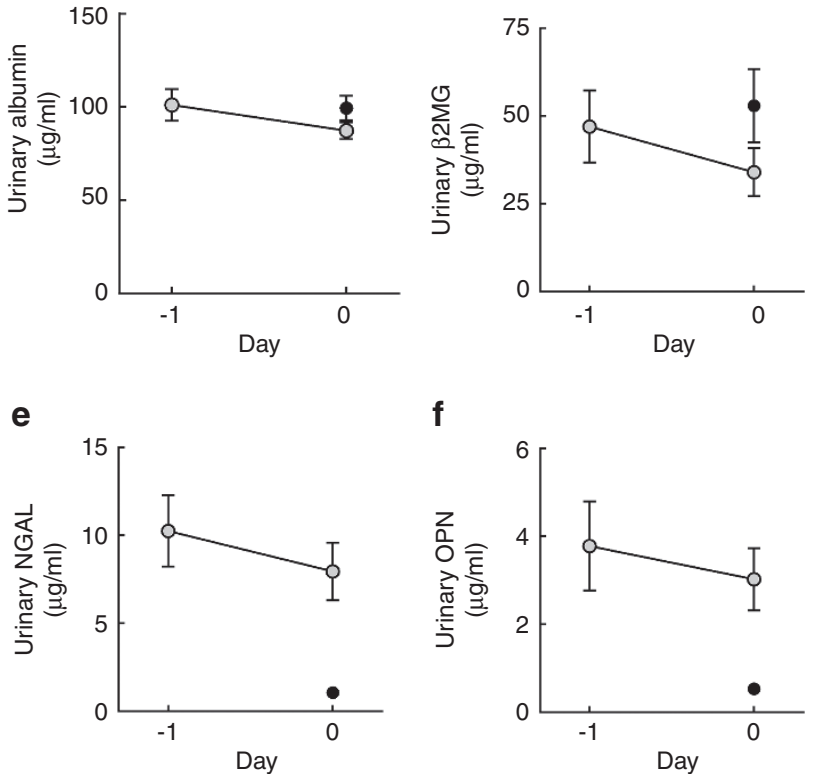

C

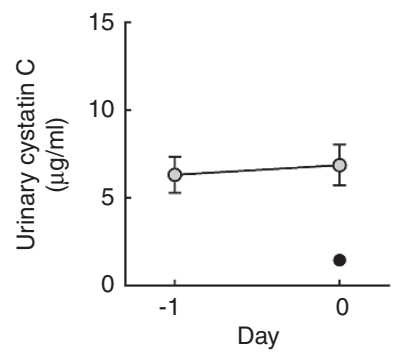

d

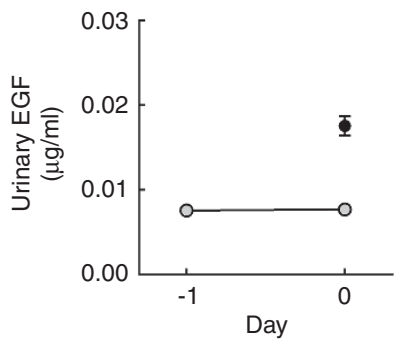

g

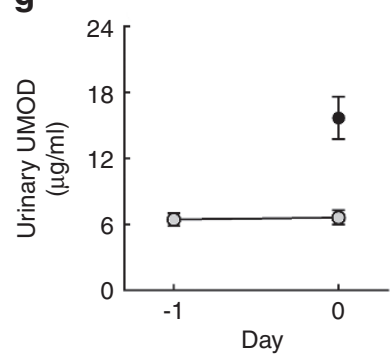

Figure 3. Urine values of Albumin, $\beta 2 M G$, Cys $C, E G F, N G A L, O P N$, and UMOD in infants with acute kidney injury (AKI) (gray circles) and infants without AKI (black circles) on day-1 and day 0. EGF, epidermal growth factor; NGAL, neutrophil gelatinase-associated lipocalin; OPN, osteopontin; UMOD, uromodulin.

Table 2. Fold change (crude) and area under the curve (adjusted model) for candidate urinary biomarkers on day -1 classified by AKI stage

\begin{tabular}{|c|c|c|c|c|c|c|}
\hline & $\begin{array}{l}\text { AKI stage I } \\
\text { fold } \\
\text { change }\end{array}$ & $P$ value & & $\begin{array}{c}\text { AKI } \\
\text { stage II/III } \\
\text { fold change }\end{array}$ & $P$ value & AUC \\
\hline Albumin $(\mu \mathrm{g} / \mathrm{ml})$ & -0.32 & 0.09 & 0.66 & -0.71 & 0.19 & 0.59 \\
\hline$\beta 2 \mathrm{MG}(\mu \mathrm{g} / \mathrm{ml})$ & -0.74 & 0.28 & 0.6 & -0.98 & 0.16 & 0.49 \\
\hline Cystatin C (ng/ml) & 1.87 & 0.002 & 0.79 & 2.86 & 0.002 & 0.82 \\
\hline $\mathrm{EGF}(\mathrm{ng} / \mathrm{ml})$ & -1.34 & $<0.001$ & 0.97 & -1.1 & 0.001 & 0.86 \\
\hline $\mathrm{NGAL}(\mathrm{ng} / \mathrm{ml})$ & 2.96 & $<0.001$ & 0.91 & 2.39 & 0.004 & 0.92 \\
\hline OPN (ng/ml) & 2.58 & 0.001 & 0.8 & 2.8 & 0.02 & 0.84 \\
\hline UMOD $(\mu \mathrm{g} / \mathrm{ml})$ & -1.24 & $<0.001$ & 0.87 & -1.22 & 0.02 & 0.85 \\
\hline
\end{tabular}

AKI, acute kidney injury; AUC, area under the curve; EGF, epidermal growth factor; NGAL, neutrophil gelatinase-associated lipocalin; OPN, osteopontin; UMOD, uromodulin.

preterm infants. The present study provides additional support for assessing urine NGAL as a biomarker of AKI in neonates in a large multicenter trial. EGF is a growth factor that stimulates cell growth, proliferation, and differentiation by binding to its receptor EGFR. EGFR activation is required for renal regeneration and functional recovery after AKI. Smith et al. (18) demonstrated that EGF signaling pathway is activated in a mouse model of sepsis-induced AKI. Chen et al. (19) reported lower urine EGF levels in infants with severe perinatal asphyxia compared with controls, suggesting that EGF may play a role in the repair of acute renal injury after asphyxia.

We controlled for birth weight as a potential confounder in evaluating the association between urine biomarkers and AKI to ensure that the detected changes in these biomarkers are

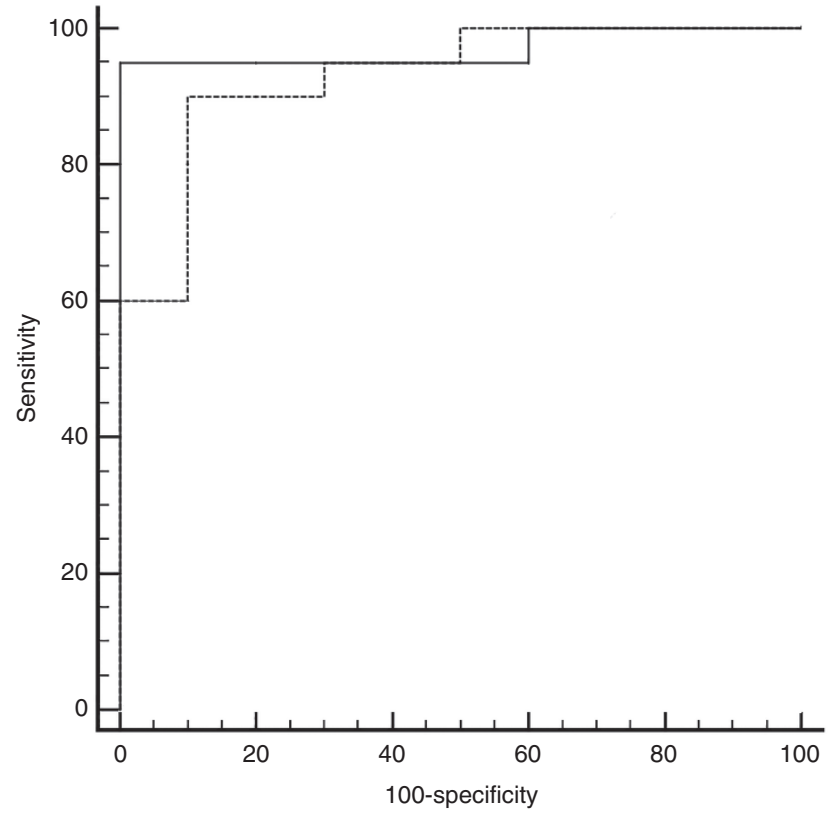

Figure 4. Receiver operating characteristics for EGF and NGAL for prediction of stage I AKI on day-1. The area under the curve is 0.97 and 0.91 respectively. (-, solid) EGF, (---, dotted) NGAL. EGF, epidermal growth factor; NGAL, neutrophil gelatinase-associated lipocalin.

not simply a reflection of prematurity (20). Askenazi et al. (21) demonstrated that urinary biomarkers can predict AKI and mortality in very-low-birth-weight infants independent of gestational age and birth weight. This study could not however determine whether these biomarkers can detect AKI before changes in SCr occur; our findings are promising and consistent with earlier literature. We could not assess the impact of 
postnatal age on urinary biomarkers. Saeidi et al. (22) showed that postnatal age affects urine biomarkers measured in the first $4 \mathrm{~d}$ of life in preterm infants without AKI. The nested casecontrol study design was not intended to assess the incidence of AKI; it has the advantage of cost and effort reduction with relatively minor loss in statistical efficiency compared with the full-cohort approach (23).

The limitations of this study are the small number and the inability to correlate between biomarkers and clinical outcomes such as duration of mechanical ventilation or mortality. However, strength of this study is the daily urine collection that allowed us to evaluate the utility of these biomarkers in the early prediction of AKI and its evaluation of seven candidate biomarkers. We found no significant difference between urinary biomarkers studied, across different gestational ages in this small cohort. Another limitation is that serum creatinine-based gold standard definition of AKI is likely not $100 \%$ accurate in detecting $\mathrm{AKI}$ in neonates, as it reflects function, rather than injury. Therefore, some control infants might have had actual kidney injury, and some infants with elevated SCr might not have had kidney damage. Our emphasis is on the potential use of changes in urine biomarkers patterns as a diagnostic tool to monitor kidney function in this vulnerable population. Future studies in larger cohorts of premature infants that involve daily urine collection to evaluate these biomarkers against hard clinical endpoints will control for these limitations and allow determining which biomarkers can best predict AKI at earlier time points.

Our findings provide new insights into the potential role of these urine biomarkers in the early prediction of AKI in this vulnerable population. Validation of these novel biomarkers with respect to preclinical and clinical use, sensitivity, and specificity will provide additional tools in the early detection of kidney injury. We are currently conducting a prospective study to develop a baseline normative renal biomarkers data set for preterm infants. In conclusion, urine biomarkers can be used as noninvasive predictors of evolving neonatal renal injury in preterm infants. Early identification of AKI prior to changes in serum creatinine allows the application of renoprotective approaches such as fluid administration management and adjustment of nephrotoxic medications in the early stages of AKI. Additionally, earlier detection of AKI might enable more rapid conventional interventions or introduction of novel therapies to prevent or effectively treat such otherwise undetected AKI.

\section{METHODS}

\section{Study Design and Patient Selection}

We conducted a prospective case-control observational study to determine the ability of seven urinary biomarkers to predict AKI prior to SCr. Using prospective data collection; infants who had AKI were identified and then controls were selected from the same cohort. The study protocol and consent forms were approved by the University of Kentucky, College of Medicine Human Subjects Institutional Review Board. Preterm infants $(<32 \mathrm{wk})$ admitted to the regional quaternary care neonatal intensive care unit of Kentucky Children's Hospital, were enrolled after obtaining parental consent. Infants with major congenital anomalies and those who did not survive to $48 \mathrm{~h}$ of life were excluded. Urine was collected once daily for the first $7 \mathrm{~d}$ of life by placing cotton balls at the perineum. Urine was extracted, centrifuged for $10 \mathrm{~min}$ to remove any cotton fibers or cellular elements, and then the supernatant was aliquoted equally into cryovials and frozen at $-80^{\circ} \mathrm{C}$ until sample evaluation.

The outcome of interest is the development of AKI. Neonatal AKI was defined according to the modified KDIGO (Kidney Disease | Improving Global Outcomes) classification described by Jetton et al., (10) each SCr is compared to the lowest previous SCr value. Day 0 was defined as the day on which the infant first met criteria for AKI, urine samples collected $24 \mathrm{~h}$ prior to day 0 (day - 1 ) were compared with control samples to evaluate the predictive value of different urinary biomarkers.

\section{Laboratory Analysis}

SCr values were obtained prospectively as part of standard patient care from the day of admission up to $14 \mathrm{~d}$ of life. Serum creatinine was measured using Jaffe method traceable to isotope dilution mass spectrometry. Biomarkers analysis was performed using Meso Scale Discovery Human Kidney Injury Panel-5 Prototype 7-Plex Assay Kit (Meso Scale Discovery, Gaithersburg, MD). Reproducibility of standard duplicates was obtained with an average signal confidence of variability of 6.5\%. The Human Kidney Injury Panel-5 (7-Plex assay) has picogram per milliliter $(\mathrm{pg} / \mathrm{ml})$ sensitivity and covers a broad concentration range, from low $\mathrm{pg} / \mathrm{ml}$ up to $200,000 \mathrm{pg} / \mathrm{ml}$. All the biomarkers levels were normalized to urinary creatinine levels. Final biomarkers values were expressed in micrograms per milliliter.

\section{Statistical Analysis}

Descriptive statistical analysis was performed to determine differences between groups. The Shapiro-Wilk test and the normal probability plot were used to test for normality of data. Normally distributed continuous variables were compared using Fisher exact test. Non-normal distributed continuous variables were analyzed using the Mann-Whitney $U$-test for two groups and Kruskal-Wallis test for multiple groups. Multiple logistic regression analysis was performed, with birth weight and AKI forced into the model, to control for birth weight as a potential confounder. For the regression analysis, biomarkers were converted to natural log to achieve normal distribution. The predictive accuracy of urine biomarkers for identifying AKI was evaluated by constructing multivariate receiver operating characteristics curves. A combination model was evaluated to examine how a combination of the two biomarkers (NGAL and EGF) could improve the ability to predict AKI. For all descriptive analysis, statistical significance was defined as an $\alpha$ value of 0.05 .

\section{ACKNOWLEDGMENTS}

We thank Vicki Whitehead and Deb Grider for assisting with patient enrollment, collection of laboratory samples, and data collection.

\section{STATEMENT OF FINANCIAL SUPPORT}

This study was supported by Children's Miracle Network.

Disclosure: Authors have no financial relationships relevant to this article to disclose.

\section{REFERENCES}

1. Howson CP, Kinney MV, McDougall L, Lawn JE; Born Too Soon Preterm Birth Action Group. Born too soon: preterm birth matters. Reprod Health 2013;10 Suppl 1:S1.

2. Parker RA, Lindstrom DP, Cotton RB. Improved survival accounts for most, but not all, of the increase in bronchopulmonary dysplasia. Pediatrics 1992;90:663-8.

3. Viswanathan S, Manyam B, Azhibekov T, Mhanna MJ. Risk factors associated with acute kidney injury in extremely low birth weight (ELBW) infants. Pediatr Nephrol 2012;27:303-11.

4. Rodríguez MM, Gómez AH, Abitbol CL, Chandar JJ, Duara S, Zilleruelo GE. Histomorphometric analysis of postnatal glomerulogenesis in extremely preterm infants. Pediatr Dev Pathol 2004;7:17-25.

5. Askenazi DJ, Koralkar R, Hundley HE, Montesanti A, Patil N, Ambalavanan N. Fluid overload and mortality are associated with acute kidney injury in sick near-term/term neonate. Pediatr Nephrol 2013;28:661-6. 
6. Koralkar R, Ambalavanan N, Levitan EB, McGwin G, Goldstein S, Askenazi D. Acute kidney injury reduces survival in very low birth weight infants. Pediatr Res 2011;69:354-8.

7. Carmody JB, Swanson JR, Rhone ET, Charlton JR. Recognition and reporting of AKI in very low birth weight infants. Clin J Am Soc Nephrol 2014;9:2036-43.

8. Abitbol CL, Bauer CR, Montané B, Chandar J, Duara S, Zilleruelo G. Longterm follow-up of extremely low birth weight infants with neonatal renal failure. Pediatr Nephrol 2003;18:887-93.

9. Auron A, Mhanna MJ. Serum creatinine in very low birth weight infants during their first days of life. J Perinatol 2006;26:755-60.

10. Jetton JG, Askenazi DJ. Update on acute kidney injury in the neonate. Curr Opin Pediatr 2012;24:191-6.

11. Askenazi DJ, Ambalavanan N, Goldstein SL. Acute kidney injury in critically ill newborns: what do we know? What do we need to learn? Pediatr Nephrol 2009;24:265-74.

12. Abitbol CL, Seeherunvong W, Galarza MG, et al. Neonatal kidney size and function in preterm infants: what is a true estimate of glomerular filtration rate? J Pediatr 2014;164:1026-1031.e2.

13. Heise D, Rentsch K, Braeuer A, Friedrich M, Quintel M. Comparison of urinary neutrophil glucosaminidase-associated lipocalin, cystatin $\mathrm{C}$, and $\alpha 1$-microglobulin for early detection of acute renal injury after cardiac surgery. Eur J Cardiothorac Surg 2011;39:38-43.

14. Herrero-Morín JD, Málaga S, Fernández N, et al. Cystatin C and beta2microglobulin: markers of glomerular filtration in critically ill children. Crit Care 2007;11:R59.
15. Zappitelli M, Washburn KK, Arikan AA, et al. Urine neutrophil gelatinaseassociated lipocalin is an early marker of acute kidney injury in critically ill children: a prospective cohort study. Crit Care 2007;11:R84.

16. Haase M, Devarajan P, Haase-Fielitz A, et al. The outcome of neutrophil gelatinase-associated lipocalin-positive subclinical acute kidney injury: a multicenter pooled analysis of prospective studies. J Am Coll Cardiol 2011;57:1752-61.

17. Sarafidis K, Tsepkentzi E, Diamanti E, et al. Urine neutrophil gelatinaseassociated lipocalin to predict acute kidney injury in preterm neonates. A pilot study. Pediatr Nephrol 2014;29:305-10.

18. Smith JA, Stallons LJ, Schnellmann RG. Renal cortical hexokinase and pentose phosphate pathway activation through the EGFR/Akt signaling pathway in endotoxin-induced acute kidney injury. Am J Physiol Renal Physiol 2014;307:F435-44.

19. Chen L, Liu W. Effect of asphyxia on urinary epidermal growth factor levels in newborns. J Tongji Med Univ 1997;17:144-6.

20. Askenazi DJ, Koralkar R, Levitan EB, et al. Baseline values of candidate urine acute kidney injury biomarkers vary by gestational age in premature infants. Pediatr Res 2011;70:302-6.

21. Askenazi DJ, Montesanti A, Hunley H, et al. Urine biomarkers predict acute kidney injury and mortality in very low birth weight infants. J Pediatr 2011;159:907-12.e1.

22. Saeidi B, Koralkar R, Griffin RL, Halloran B, Ambalavanan N, Askenazi DJ. Impact of gestational age, sex, and postnatal age on urine biomarkers in premature neonates. Pediatr Nephrol 2015;30:2037-44.

23. Ernster VL. Nested case-control studies. Prev Med 1994;23:587-90. 